\title{
COUPLED STATES OF THE SYSTEM OF TWO FREE ELECTRONS ON A SINGLE STAR *
}

\author{
S. WALCERZ AND T. LULEK \\ A. Mickiewicz University, Institute of Physics, \\ Matejki 48/49, 60-769 Poznań, Poland \\ (Received July 15, 1991; in final form September 6, 1991)
}

\begin{abstract}
A classification scheme of quantum states for the system. of two free electrons in a cubic box, confined to a single star of quasi-momentum is proposed within a Racah-Wigner type of approach. Coupling of angular momenta of the atomic case is here substituted by Mackey theorem for transitive representations, which provides a crystalline analogue of orbital angular momentum - the resultant orbit of the geometric symmetry group. The action of the Pauli group is combined with that of the octahedral group which yields the connection between spin (i.e. the singlet or triplet pairing of electrons) and statistic of the positional factor. Resultant singlets and doublets - irreducible representations of the octahedral group, exhibit an ordinary Landau diamagnetic behaviour, whereas triplets are paramagnetic. A relation between the Mackey star and the star of resultant momentum is discussed.
\end{abstract}

PACS numbers: 02.20.+b, 71.45.-d, 75.10.Lp, 75.20.-g

\section{Introduction}

The aim of the present paper is to propose a classification of states of the system consisting of two free electrons in a cubic box. Moreover, we assume that the space of quantum states of a single electron is confined to a single star of a wavevector in the reciprocal space. The single-electron space is thus spanned on a small number of de Broglie electron waves, corresponding to the same energy. This space is also closed under the action of the octahedral group $O_{h}$ - the geometric symmetry group of the box.

Such a problem is one of the initial steps in an adaptation of Racah-Wigner type of approach to description of electronic structure in solids within the

*The work has been supported by the Ministry of Education research project $\mathrm{P} / 04 / 250 / 90-2 / \mathrm{V} / 1-9$. 
free-electron model (Lulek [1]). Quantization of momentum implies discreteness of the energy spectrum, and a single star in the Brillouin zone is a plausible crystalline analogue of $n l$ electronic shell in a atom, at least in cases of mesoscopic regions. In this paper we examine the freedom of construction of symmetry adapted coupled two-electron states within a single star, in a way resemblying coupling of two angular momenta in atomic theory. The spherical symmetry is substituted by the octahedral one, and coupling of angular momenta, i.e. decomposition of irreducible representations of the SU2 group by Mackey theorem for transitive representations of the group $O_{h}$, acting on appropriate stars in the Brillouin zone.

\section{The single-electron space}

$$
\begin{aligned}
& \text { Let } \\
& \gamma=\left\{g k \mid g \in O_{h}\right\}
\end{aligned}
$$

be the star, generated by the octahedral group $O_{h}$ from the wave vector

$$
k=\frac{2 \pi}{Q a}(p, q, r) \equiv\left(k_{1}, k_{2}, k_{3}\right),
$$

where $Q a$ is the length of the edge of the cubic box confining electrons, $p, q, r$ are non-negative integers, and the cartesian coordinate system in (2) coincides with fourfold axes of the group $O_{h}$. The linear unitary space over the field $\mathcal{C}$ of complex numbers, spanned on the star $\gamma$ as its orthonormal basis, i.e.

$$
L_{0}=\operatorname{lc}_{\mathcal{C}} \gamma
$$

where lc denotes the linear closure, will be referred to as the single-electron orbital space. It is the carrier space of the linear complement of the transitive representation $R^{O_{h}: D}$ of the group $O_{h}$, acting on the star $\gamma$ as its orbit, with the subgroup

$$
D=\left\{g \in O_{h} \mid g k=k\right\} \subset O_{h}
$$

being the stabiliser of the wave vector $k$.

The single-electron space of our problem is the tensor product

$$
L=L_{0} \otimes L_{s}
$$

of the orbital space $L_{0}$ by the standard two-dimensional spin space $L_{s}$ - the carrier space of the irreducible representation $D^{1 / 2}$ of the SU2 group. Evidently,

$$
\operatorname{dim} L=2 \operatorname{dim} L_{0}=2|\gamma|,
$$

where $|\gamma|$ is the number of elements (arms) in $\gamma$. The set

$$
b_{\text {in }}(L)=\{|k \sigma\rangle \mid k \in \gamma, \quad \sigma \in \tilde{s}=\{ \pm 1 / 2\}\}
$$

serves as an initial orthonormal basis in $L$. Another basis emerges from the decomposition

$$
R^{O_{h}: D} \approx \sum_{\Lambda \in \widetilde{O}_{h}} \oplus m(D, \Lambda) \Lambda
$$


of the transitive representation $R^{O_{h}: D}$ into irreducible representations $A$ of the octahedral group, with $\widetilde{O}_{h}$ being the set of all irreducible representations of $O_{h}$, and $m(D, \Lambda)$ the corresponding multiplicities. This irreducible basis is written as

$$
b_{\text {irr }}(L)=\left\{|\Lambda t \lambda s\rangle \mid \Lambda \in \tilde{O}_{h}, t \in \tilde{m}(D, \Lambda), \lambda \in \tilde{\Lambda}, \sigma \in \tilde{s}\right\},
$$

where $t \in \tilde{m}(D, \Lambda)$ is the repetition label for $\Lambda$, and $\lambda \in \tilde{\Lambda}$ is a standard basis function of $\Lambda$.

In order to arrive at a clear scheme of classification, we shortly restate these notions in terms of "action of a group on a set" ([Michel [2]). Let

$$
\mathcal{T}: O_{h} \times \mathcal{Z}^{3} \longrightarrow \mathcal{Z}^{3}
$$

be the action of the octahedral group $O_{h}$ on the set $\mathcal{Z}^{3}$ of all wave vectors $k$ in the reciprocal space. Then every star $\gamma$ given by Eq. (1) is an orbit of this action, characterized by the conjugacy class (epikernel)

$$
[D]=\left\{g D g^{-1} \mid g \in O_{h}\right\}
$$

of the stabiliser $D$. Let $\mathcal{Z}^{3} / \mathcal{T}$ denote the set of all orbits of $\mathcal{T}$. The subset

$$
\mathcal{S}[D]=\left\{\gamma \in \mathcal{Z}^{3} / \mathcal{T} \mid[D(\gamma)]=[D]\right\}
$$

of all orbits with the same epikernel $[D]$ constitutes the stratum. Decomposition

$$
\mathcal{Z}^{3} / \mathcal{T}=\bigcup_{[D] \in e k T} \mathcal{S}[D]
$$

of the set $\mathcal{Z}^{3} / \mathcal{T}$ of all orbits into strata $\mathcal{S}[D]$ is called the stratification of $\mathcal{Z}^{3}$ under the action $\mathcal{T}$, and the set $e k \mathcal{T}$ of all epikernels with non-empty strata is a subset of the lattice $\tilde{l}\left(O_{h}\right)$ of all conjugacy classes of subgroups of the octahedral group $O_{h}$. Following Newman [3], we denote each stratum of $\mathcal{T}$ by a sequence $(p q r)$ of three non-negative integers, some of them could be equal to another or to zero. The lattice $e k \mathcal{T}$ is given in Fig. 1, and the details of stratification are listed in Table I.

\section{TABLE I}

Stratification of the set $\mathcal{Z}^{3}$ of all vectors admissible by quantization conditions, under the action $\mathcal{T}$ of the octahedral group $O_{h}$.

\begin{tabular}{c|c|r|l}
\hline $\begin{array}{c}\text { The generator } \\
\text { of a star } \gamma\end{array}$ & The stabiliser & $|\gamma|$ & $\begin{array}{l}\text { Dimension } \\
\text { of stratum in } R^{3}\end{array}$ \\
\hline$p q r$ & $C_{1}$ & 48 & 3 (generic) \\
$p q 0$ & $C_{1 h}$ & 24 & 2 \\
$p p q$ & $C_{1 h}^{\prime}$ & 24 & 2 \\
$p p 0$ & $C_{2 v}^{\prime}$ & 12 & 1 \\
$p p p$ & $C_{3 v}$ & 8 & 1 \\
$p 00$ & $C_{4 v}$ & 6 & 1 \\
000 & $O_{h}$ & 1 & 0
\end{tabular}




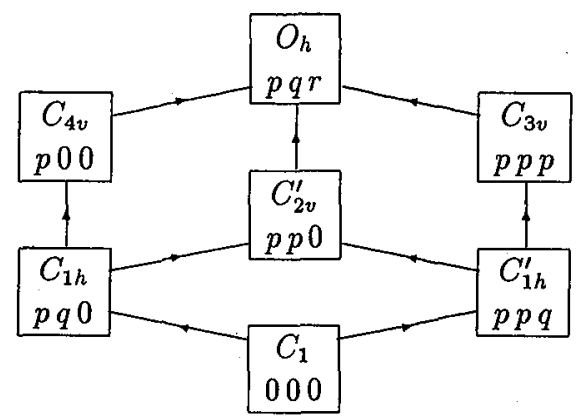

Fig. 1. The lattice $e k \mathcal{T}$ of epikernels.

\section{The two-electron space}

The space of two-electron states is the antisymmetric part $L\left\{1^{2}\right\}$ of the tensor product $L \otimes L$. The classification problem consists in a construction of an orthonormal basis in $L\left\{1^{2}\right\}$.

We can proceed essentially in the same way as described by Lulek [3] and Lulek and Szopa [4] for the localised case. Perhaps the simplest is the choice of non-correlated states, i.e. Slater determinants of basic functions (7). Such a basis corresponds to an ordinary second-quantized method which is insensitive to any electron correlations. Another choice, the irreducible scheme of [2] starts from the irreducible basis (9), and couples the orbital single-electron states $\Lambda_{1}$ and $\Lambda_{2}$ as

$$
\Lambda_{1} \otimes \Lambda_{2}=\sum_{\Lambda \in \widetilde{O}_{h}} \oplus m\left(\Lambda_{1} \otimes \Lambda_{2}, \Lambda\right) \Lambda
$$

into two-electron orbital manifolds $A$. This choice takes into account the factorization (5) of the single-electron space into orbital and spin factors and yields the corresponding quantum numbers $A$ and $S$ - counterparts of atomic $L S$ numbers for the system of $N=2$ electrons, in a spirit of Wigner-Racah approach. The main disadvantage of this choice is that it is able to account only for a narrow variety of correlations effective within manifolds $\Lambda_{1}$ and $\Lambda_{2}$, leaving aside the rest of the shell defined by the star $\gamma$.

This disadvantage can be remedied by use of the transitive scheme of papers [3-4], where the permutational structure of the orbital representation $R^{O_{h}: D}$ can be exploited by Mackey theorem [5]. This theorem is related to a fibration of the cartesian square $\gamma \times \gamma$ of the single-electron star $\gamma$, consistent with the action $R^{O_{h}: D} \times R^{O_{h}: D}$ of the octahedral group $O_{h}$. In spite of its implications in classification procedures, and its parallelism to coupling of angular momenta, we consider this fibration in some more details in the next chapter. 


\section{A fibration imposed by Mackey theorem}

The Mackey fibration can be performed in two steps. Firstly we consider the subduction $\left(R^{O_{h}: D} \times R^{O_{h}: D}\right) \downarrow D$, and restrict it to the first edge of the square $\gamma \times \gamma$, i.e. to the set

$$
\gamma^{1}=\left\{|g k, k\rangle \mid g \in O_{h}\right\} \subset \gamma \times \gamma,
$$

where $k \in \gamma$ is a wave vector of the star $\gamma$ with the stabiliser $D$. Clearly, the orbit generated from $|g k, k\rangle$ is

$$
\mathcal{O}_{D}[|g k, k\rangle]=\{|d g k, d k\rangle \mid d \in D\},
$$

with the stabiliser

$$
L(\mid g k, k))=D \cap g D g^{-1} \text {. }
$$

Moreover, each element $g^{\prime} \in D g D$ generates the same orbit (15) as $g$, so these orbits are classified by the double cosets of $G$. Let

$$
O_{h}=\bigcup_{\omega \in \Omega} D g_{\omega} D
$$

be the decomposition of the group $O_{h}$ into double cosets $\omega \in \Omega$, with arbitrarily chosen representatives $g_{\omega}$. Then the considered subduction, restricted to the first edge $\gamma^{1}$, decomposes into transitive representations of the stabiliser $D$ as

$$
\left.\left(R^{O_{h}: D} \times R^{O_{h}: D}\right) \downarrow D\right|_{\gamma^{1}}=\sum_{\omega \in \Omega} R^{D: L_{\omega}},
$$

where

$$
L_{\omega}=D \cap g_{\omega} D g_{\omega}^{-1}
$$

and the set $\Omega$ is defined by Eq. (17).

In the second step we make the identification

$$
R^{O_{h}: D} \times R^{O_{h}: D}=\left(\left.\left(R^{O_{h}: D} \times R^{O_{h}: D}\right) \downarrow D\right|_{\gamma^{1}}\right) \uparrow O_{h},
$$

i.e. induce the representation (18) of the subgroup $D \subset O_{h}$ to the group $O_{h}$. The orbit

$$
\mathcal{O}[|g k, k\rangle]=\left\{|x g k, x k\rangle \mid x \in O_{h}\right\}
$$

of the group $O_{h}$ can be looked at as a bundle with the base (15) and the typical fiber $\gamma$. Each such bundle defines an orbit of the $O_{h}$ group, labelled by $\omega \in \Omega$. Elements of the base $\mathcal{O}_{D}[|g k, k\rangle]$ of this bundle are labelled by the index $\lambda$, defined by the decomposition

$$
D=\bigcup_{\lambda \in \widetilde{R}\left(D: L_{\omega}\right)} d_{\lambda} L_{\omega}
$$


of the group $D$ into the left cosets with respect to the stabiliser $L_{\omega}$, and the elements of the fiber $\left\{g_{r_{2}} d_{\lambda} L_{\omega} \mid r_{2} \in \tilde{R}\right\}$ over $\lambda$ are labelled by the index $r_{2}$, denoting the second argument of the ket

$$
\left|k_{1}, k_{2}\right\rangle=|x g k, x k\rangle=\left|g_{r_{1}} k, g_{r_{2}} k\right\rangle \in \gamma \times \gamma
$$

The fibration $\xi$ of the cartesian square $\gamma \times \gamma$ yields thus the substitution

$$
\xi\left(r_{1}, r_{2}\right)=\left(\omega, \lambda, r_{2}\right), \quad\left(r_{1}, r_{2}\right) \in \gamma \times \gamma
$$

of cartesian coordinates $\left(r_{1}, r_{2}\right)$ by fibre ones $\left(\omega, \lambda, r_{2}\right)$. In another notation, we have

$$
\xi(|x g k, x k\rangle)=\left|g_{\lambda} g_{\omega} k, g_{r_{2}} k\right\rangle, \quad x \in O_{h}, \quad g \in O_{h}
$$

where $g_{\omega}, g_{\lambda}$ and $g_{r_{2}}$ are uniquely defined by selection rules

$$
g_{\omega} \in D x g D, \quad g_{\lambda} g_{\omega} \in x g D, \quad g_{r_{2}} \in x D
$$

The fibration $\xi$ can be presented schematically on Fig. 2. An example of this fibration for the star $\gamma=p 00$ is given in Table II.

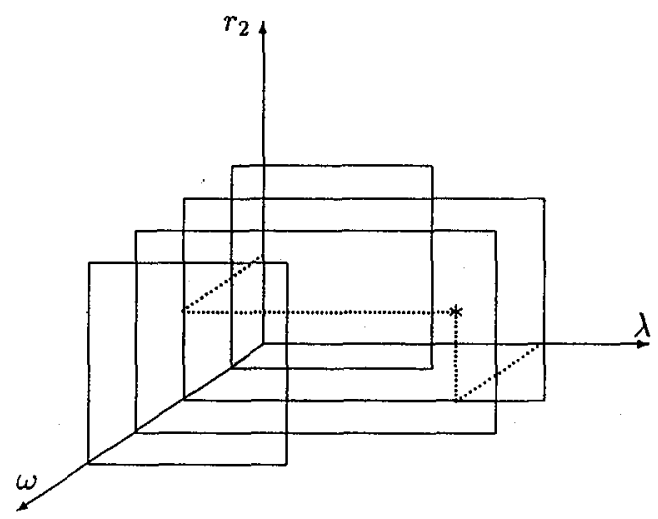

Fig. 2. Fibration of $\gamma \times \gamma=\left\{\left(r_{1}, r_{2}\right) \in \gamma\right\}$. Each sheet $\omega$ denotes an orbit of the octahedral group. This sheet is presented here as a bundle with the base lying along the $\lambda$-axis, and fibers along $r_{2}$-axis. Observe that various bundles $\omega$ can differ by their bases, but all have isomorphic fibres. An element $\left(r_{1}, r_{2}\right) \in \gamma \times \gamma$, denoted by the asterisk, achieves the fibre coordinates $\xi\left(r_{1}, r_{2}\right)=\left(\omega, \lambda, r_{2}\right)$. 
TABLE II

The fibration on the square $\gamma \times \gamma$ for the star $\gamma=p 00$. The symbols $\left(\omega, \lambda, r_{2}\right)$ are fibre coordinates of $\left(r_{1}, r_{2}\right) \in \gamma \times \gamma ; \omega$ and $\lambda$ are represented respectively by $g_{\omega}$ and $g_{\lambda}$. We identify here $g_{\lambda}=d_{\lambda}$ for clarity, and omit $r_{2}$ coordinate since it is the same for all states in the fiber $(\omega, \lambda)$.

\begin{tabular}{c|c|c|l}
\hline$\omega$ & $\lambda$ & $d_{\lambda}$ & \multicolumn{1}{c}{$r_{1}$} \\
\hline$E$ & $E$ & $E, C_{4 x}^{+}, C_{2 x}, C_{4 x}^{-}, \sigma_{y}, \sigma_{z}, \sigma_{A B}, \sigma_{C D}$ & $(p, 0,0)$ \\
\hline$C_{3 A}^{+}$ & $E$ & $E, \sigma_{y}$ & $(0,0, p)$ \\
& $C_{4 x}^{+}$ & $C_{4 x}^{+}, \sigma_{C D}$ & $(0, p, 0)$ \\
& $C_{2 x}$ & $C_{2 x}, \sigma_{z}$ & $(0,0,-p)$ \\
& $C_{4 x}^{-}$ & $C_{4 x}^{-}, \sigma_{A B}$ & $(0,-p, 0)$ \\
\hline$C_{3 A}^{-}$ & $E$ & $E, \sigma_{z}$ & $(0, p, 0)$ \\
& $C_{4 x}^{+}$ & $C_{4 x}^{+}, \sigma_{A B}$ & $(0,0,-p)$ \\
& $C_{2 x}$ & $C_{2 x}, \sigma_{y}$ & $(0,-p, 0)$ \\
& $C_{4 x}^{-}$ & $C_{4 x}^{-}, \sigma_{C D}$ & $(0,0, p)$ \\
\hline$u_{B C}$ & $E$ & $E, \sigma_{z}$ & $(0,-p, 0)$ \\
& $C_{4 x}^{+}$ & $C_{4 x}^{+}, \sigma_{A B}$ & $(0,0, p)$ \\
& $C_{2 x}$ & $C_{2 x}, \sigma_{y}$ & $(0, p, 0)$ \\
& $C_{4 x}^{-}$ & $C_{4 x}^{-}, \sigma_{C D}$ & $(0,0,-p)$ \\
\hline$u_{B D}$ & $E$ & $E, \sigma_{y}$ & $(0,0,-p)$ \\
& $C_{4 x}^{+}$ & $C_{4 x}^{+}, \sigma_{C D}$ & $(0,-p, 0)$ \\
& $C_{2 x}$ & $C_{2 x}, \sigma_{z}$ & $(0,0, p)$ \\
& $C_{4 x}^{-}$ & $C_{4 x}^{-}, \sigma_{A B}$ & $(0, p, 0)$ \\
\hline$u_{B C}$ & $E$ & $E, C_{4 x}^{+}, C_{2 x}, C_{4 x}^{-}, \sigma_{y}, \sigma_{z}, \sigma_{A B}, \sigma_{C D}$ & $(-p, 0,0)$
\end{tabular}

\section{Coupling of momenta}

Globally, the stratification of the square $\gamma \times \gamma$ under the action $\mathcal{T}^{2}$ of the octahedral group $O_{h}$ can be written as

$$
R^{O_{h}: D} \times R^{O_{h}: D}=\sum_{L \in e k T} m(D, L) R^{O_{h}: L}
$$

where $m(D, L)$ is the multiplicity of the transitive representation $R^{O_{h}: L}$ in $R^{O_{h}: D} \times$ $R^{O_{h}: D}$. These multiplicities are listed in Table III. 
TABLE III

The multiplicities $m(D, L)$ of resultant transitive representations $R^{O_{h}: L}$ in $R^{O_{h}: D} \times R^{O_{h}: D}$, i.e. the number of orbits with the epikernel $L$ on the square $\gamma \times \gamma$.

\begin{tabular}{l|c|c|c|c|c|c|c}
\hline \hline$D$ & \multicolumn{10}{|c}{$L$} \\
\hline & $C_{1}$ & $C_{1 h}$ & $C_{1 h}^{\prime}$ & $C_{2 v}^{\prime}$ & $C_{3 v}$ & $C_{4 v}$ & $O_{h}$ \\
\hline$C_{1}$ & 48 & & & & & & \\
$C_{1 h}$ & 8 & 8 & & & & & \\
$C_{1 h}^{\prime}$ & 10 & & 4 & & & & \\
$C_{2 v}^{\prime}$ & 2 & 1 & & 2 & & & \\
$C_{3 v}$ & & & 2 & & 2 & & \\
$C_{4 v}$ & & 1 & & & & 2 & \\
$O_{h}$ & & & & & & & 1
\end{tabular}

The resultant momentum $K$ of the system of two electrons in the state $\left|g_{\omega} k, k\right\rangle$ is

$$
K=g k+k .
$$

Let $D(K)$ be the stabiliser of $K$, in accordance with Eq. (4). Clearly,

$$
L_{\omega} \subseteq D(\boldsymbol{K}),
$$

i.e. the stabiliser of $K$ encloses the stabiliser $L_{\omega}$ of the Mackey orbit $\omega$. However, the inclusion $\subseteq$ in Eq. (28) cannot be substituted, in general, by equality since the wave vector $K$ can achieve some more symmetrical position in $\mathcal{Z}^{3}$ due to the structure of module. E.g., the pair $|-\mathbf{k}, k\rangle$ of opposite momenta belongs to the Mackey orbit with

$$
L_{\omega}=D(k) \cap D(-k)=D(k) .
$$

whereas

$$
D(\boldsymbol{K})=D(0)=O_{h},
$$

so that $L_{\omega}$ is a proper, nontrivial subgroup of $D(\boldsymbol{K})$.

In general, the Mackey orbit $\omega$ can be divided into $48 /|D(K)|$ imprimitivity sets, each having $|D(K)| /\left|L_{\omega}\right|$ two-electron states with the same resultant quasi-momentum. These imprimitivity sets are related to the chain of subgroups

$$
L_{\boldsymbol{\omega}} \subset D(\boldsymbol{K}) \subset O_{h} .
$$

E.g. in the case of a regular orbit, $\gamma=p q r,[D]=C_{1}$, each $g \in O_{h}$ is a double coset itself, and generates a regular two-electron orbit $\left\{|x g k, x k\rangle \mid x \in O_{h}\right\}$. The stabiliser of the resultant quasi-momentum depends then only upon the class of 
$O_{h}$, namely

$$
D(g k+k)= \begin{cases}C_{1} & \text { for } g \in\{E\},\left\{8 C_{3}\right\},\left\{6 C_{4}\right\},\left\{8 S_{6}\right\}, \\ C_{1 h} & \text { for } g \in\left\{6 S_{4}\right\},\{3 \sigma\} \\ C_{1 h}^{\prime} & \text { for } g \in\left\{6 \sigma^{\prime}\right\} \\ C_{2 v}^{\prime} & \text { for } g \in\{6 u\} \\ C_{4 v} & \text { for } g \in\left\{3 C_{2}\right\} \\ O_{h} & \text { for } g=I\end{cases}
$$

\section{Symmetrization}

The Pauli group

$\Sigma_{2}=\{e, t\}$

where $e$ is the unit permutation and $t-$ the transposition of electrons, realises the symmetrization related to indistinguishability of electrons. The action

$$
\mathcal{P}_{0}: \Sigma_{2} \times L_{0}^{2} \longrightarrow L_{0}^{2}
$$

of the Pauli group $\Sigma_{2}$ in the orbital space $L_{0}^{2}$ is determined by

$$
\mathcal{P}_{0}(t)=\left(\begin{array}{l}
\left|k_{1}, k_{2}\right\rangle \\
\left|k_{2}, k_{1}\right\rangle
\end{array}\right), \quad\left|k_{1}, k_{2}\right\rangle \in \gamma \times \gamma .
$$

Clearly, this action commutes with the geometric action $\mathcal{T}^{2}: O_{h} \times L_{0}^{2} \longrightarrow L_{0}^{2}$, imposed by the action $\mathcal{T}$ on the star $\gamma$, i.e.

$$
\mathcal{P}_{0}(t) \mathcal{T}^{2}(g)=\mathcal{T}^{2}(g) \mathcal{P}_{0}(t), g \in O_{h} .
$$

In other words, these actions mutually centralise, as a particular case of Weyl's duality [6] between linear and symmetric groups in tensor spaces. It allows us to use $\mathrm{S}$ irreducible representations of both groups as compatible quantum numbers for classification of two-electron states.

Let $\left|g_{\omega} k, k\right\rangle$ be the representative of an orbit of the group $O_{h}$ on the square $\gamma \times \gamma$. Then we have

$$
\mathcal{P}_{0}\left|g_{\omega} k, k\right\rangle=\left|k, g_{\omega} k\right\rangle \in \mathcal{O}\left[\left|g_{\omega}^{-1} k, k\right\rangle\right],
$$

i.e. the transposition of electrons causes a jump from the orbit $\omega$ of $O_{h}$ to the orbit determined by the inverse double coset $D g_{\omega}^{-1} D$. Simple formal properties of double cosets (Frame [7]; cf. also [3]) imply that there are two possible cases: (i) The orbit is reflexive, i.e. $D g_{\omega} D=D g_{\omega}^{-1} D$ - then it is closed under the action $\mathcal{P}_{0}$ of $\Sigma_{2}$; (ii) the orbit is not reflexive, $D g_{\omega}^{-1} D \cap D g_{\omega} D=\emptyset$ - then the orbit is not closed under the action $\mathcal{P}_{0}$, but the pair of orbits $D g_{\omega} D$ and $D g_{\omega}^{-1} D$ is, so that the Pauli group traverses this pair. Moreover, it is convenient to distinguish, within the case (i), the diagonal orbit $\mathcal{O}[|k, k\rangle]$, since the action $\mathcal{P}_{0}$ of the Pauli group generates there only invariants, i.e. single-element orbits. In other cases the 
orbits of $\mathcal{P}_{0}$ are regular, i.e. they have two elements. If we thus decompose the set $\Omega$ of orbits of $O_{h}$ in $\gamma \times \gamma$ into disjoint subsets

$$
\Omega=\Omega_{d} \cup \Omega_{r} \cup \Omega_{n},
$$

where $\Omega_{d}$ consists of the single diagonal orbit, and $\Omega_{r}$ and $\Omega_{n}$ denotes respectively nondiagonal reflexive and non-reflexive orbits, then each subset in (36) is invariant under the action $\mathcal{P}$ of the Pauli group $\Sigma_{2}$. Accordingly, the set of orbits of $\Sigma_{2}$ on $\Omega$ can be written as

$$
\Omega / \mathcal{P}_{0}=\Omega_{d} \cup \Omega_{r} \cup \Omega_{n} / 2 .
$$

Clearly, this set labels also orbits of the direct product group $O_{h} \times \Sigma_{2}$ on $\gamma \times \gamma$.

Let $L_{|\omega|}$ be the space spanned on an orbit of $O_{h} \times \Sigma_{2}$ on $\gamma \times \gamma$, i.e.

$$
L_{|\omega|}= \begin{cases}\operatorname{lc}_{\mathcal{C}}\left\{|x k, x k\rangle\left|x \in O_{h}\right\rangle\right\} & \text { for }|\omega| \in \Omega_{d}, \\ \operatorname{lc}_{\mathcal{C}}\left\{\left|x g_{\omega} k, x k\right\rangle\left|x \in O_{h}\right\rangle\right\} & \text { for }|\omega| \in \Omega_{r}, \\ \operatorname{lc}_{\mathcal{C}}\left\{\left|x g_{\omega} k, x k\right\rangle,\left|x g_{\omega}^{-1} k, x k\right\rangle\left|x \in O_{h}\right\rangle\right\} & \text { for }|\omega| \in \Omega_{n} / 2\end{cases}
$$

This space is closed under the actions of both octahedral group $O_{h}$ and Pauli group $\Sigma_{2}$, and can be decomposed into subspaces irreducible under both groups. Thus we arrive at the following orthonormal basis for the orbital space $L_{0}^{2}$

$$
\begin{array}{r}
b\left(L_{0}^{2}\right)=\left\{\left|\gamma^{2} \omega y_{0} \Lambda t \lambda\right\rangle|| \omega \mid \in \Omega / \mathcal{P}_{0}, y_{0} \in \widetilde{\Sigma}_{2},\right. \\
\left.\Lambda \in \widetilde{O}_{h}, t \in \widetilde{m}\left(|\omega|, y_{0}, \Lambda\right), \lambda \in \tilde{\Lambda}\right\},
\end{array}
$$

where $\widetilde{\Sigma}_{2}=\left\{\{2\},\left\{1^{2}\right\}\right\}$ is the set of Young diagrams for the symmetric group $\Sigma_{2}$, and $\widetilde{m}\left(|\omega|, y_{0}, \Lambda\right)$ denotes the set of repetition labels for $\Lambda$ in the space $L_{|\omega|}^{y_{0}}$, i.e. the subspace of $L_{|\omega|}$, symmetrized according to the Young diagram $y_{0}$.

For the two-electron spin space $L_{s}^{2}$ we have

$$
L_{s}^{2}=\sum_{y_{s} \in \widetilde{\Sigma}_{2}} L_{s}^{y_{s}} \equiv L_{s}^{\{2\}} \oplus L_{s}^{\left\{1^{2}\right\}},
$$

where $L_{s}^{\{2\}}$ is the three-dimensional space corresponding to the total spin $S=1$ (triplet pairing), and $L_{s}^{\left\{1^{2}\right\}}$ is the one-dimensional space with $S=0$ (singlet pairing). The Pauli exclusion principle demands

$$
y_{0} \otimes y_{s}=\left\{1^{2}\right\},
$$

where $\otimes$ denotes the inner (Clebsch-Gordan) product of irreducible representations of the Pauli group $\Sigma_{2}$. Thus we have

$$
L^{\left\{1^{2}\right\}}=\left(L_{0}^{\{2\}} \otimes L_{s}^{\left\{1^{2}\right\}}\right) \oplus\left(L_{0}^{\left\{1^{2}\right\}} \otimes L_{s}^{\{2\}}\right),
$$

which implies that $y_{0}=\{2\}$ is associated with $S=0$, whereas $y_{0}=\left\{1^{2}\right\}$ corresponds to $S=1$. Finally, the orthonormal basis for the total space $L\left\{1^{2}\right\}$ has the 
form

$$
\begin{gathered}
b\left(L\left\{1^{2}\right\}\right)=\left\{\left|\gamma^{2} \omega y_{0} \Lambda t \lambda, S M\right\rangle|| \omega \mid \in \Omega / \mathcal{P}_{0}, y_{0} \in \widetilde{\Sigma}_{2}, \Lambda \in \widetilde{O}_{h},\right. \\
\left.t \in \tilde{m}\left(|\omega|, y_{0}, \Lambda\right), \lambda \in \tilde{\Lambda}, S=0(1) \text { for } y_{0}=\{2\}\left(\left\{1^{2}\right\}\right), M \in \widetilde{S}\right\}
\end{gathered}
$$

with $M$ being the projection of the total spin, i.e. $\widetilde{S}=\{0, \pm 1\}$ for $S=1$ and $\widetilde{S}=\{0\}$ for $S=0$.

\section{Discussion}

We have proposed a classification scheme for coupled basis states of the system of two free electrons in a cubic box, within a single star $\gamma$ of one-electron states. This scheme is described by the states

$$
\left|\gamma^{2},\right| \omega\left|\Lambda t \lambda, S M_{S}\right\rangle
$$

which are some crystalline analogues of atomic states

$$
\left|l^{N}, \alpha L M_{L}, S M_{S}\right\rangle
$$

for the case of $N=2$ electrons. Thus the star $\gamma^{N}$ works as the counterpart of electronic configuration $l^{N}, \omega \Lambda t \lambda$ correspond to atomic $\alpha L M_{L}$ orbital labels, and $S M_{S}$ are quantum numbers describing the total spin. We proceed to discuss the relevance of these new labels in some more details.

The label $|\omega|$ emerges as the Mackey orbit on the Cartesian square $\gamma \times \gamma$ and describes the class of geometrically equivalent pairs $\left(k, g_{\omega} k\right)$ of quasi-momenta of electrons. It is thus related to the total quasi-momentum of the system. Moreover, this label involves the action $\mathcal{P}_{0}$ of the Pauli group which encompasses the interplay between the geometric action $\mathcal{T}^{2}$ and the requirement of indistinguishability involved in $\mathcal{P}_{0}$.

The proposed scheme is factorized, i.e. each basis state is a tensor product of positional and spin factors, in a full analogy to atomic theory. Such a scheme involves, however, a definite connection between spin and statistic of the positional factor. Thus the case $S=0$, i.e. the singlet pairing, is associated with symmetric positional states $\left(y_{0}=\{2\}\right)$, whereas the case $S=1$, i.e. the triplet pairing with antisymmetric positional statistic $\left(y_{0}=\left\{1^{2}\right\}\right)$. It is reflected in the range of the repetition label $t$ for an irreducible representation $\Lambda$ of $O_{h}$ in $|\omega|$. This label runs over all symmetric (antisymmetric) values for $S=0(1)$.

Two-electron states have a large degree of freedom, allowing for a variety of magnetic properties of their positional part. This freedom is reflected in the dimension $|\gamma|^{2}$ of the two-particle positional space, spanned on the square $\gamma \times \gamma$ of the star $\gamma$. This dimension varies from $6 \times 6=36$ for the star $p 00$ to $48 \times 48=2304$ for the regular star $p q r$.

The action $\mathcal{T}^{2}$ of the octahedral group in these spaces yields the decomposition into irreducible representations $\Lambda \in \widetilde{O_{h}}$ and appropriate irreducible subspaces, with definite rotational properties. In general, singlets $\left(\Lambda=A_{1 g}, A_{1 u}, A_{2 g}, A_{2 u}\right)$ 
and doublets $\left(E_{g}, E_{u}\right)$ yield diamagnetic properties, which are similar to Landau diamagnetism of the gas of free electrons within the single-electron picture, whereas triplets $\left(T_{1 g}, T_{1 u}, T_{2 g}, T_{2 u}\right)$ are associated with some paramagnetic properties, as in an ordinary crystal field description. Thus the gas of free electrons can exhibit some remarkable paramagnetism resulting from partial filling of a degenerate Fermi level.

\section{References}

[1] T. Lulek, Acta Phys. Pol., A80, 769 (1991).

[2] L. Michel, Rev. Mod. Phys. 52, 617 (1980).

[3] T. Lulek, Acta Phys. Pol. A75, 487 (1989).

[4] T. Lulek, M. Szopa, J. Phys. A, Math. Gen. 23, 677 (1990).

[5] B. Lulek, T. Lulek, R. Chatterjee, J. Biel, Can. J. Phys. 63, 1061 (1985).

[6] H. Weyl, The Theory of Groups and Quantum Mechanics, Dover, New York 1950.

[7] J.S. Frame, Bull. Am. Math. Soc. 47, 458 (1941). 